\title{
Fatigue in early, intensively treated and tight-controlled rheumatoid arthritis patients is frequent and persistent: a prospective study
}

\author{
Margot J. M. Walter ${ }^{1} \cdot$ T. M. Kuijper ${ }^{1,2} \cdot$ J. M. W. Hazes ${ }^{1} \cdot$ A. E. Weel ${ }^{1,2} \cdot$ J. J. Luime ${ }^{1}$
}

Received: 10 April 2018 / Accepted: 13 July 2018 / Published online: 16 July 2018

(c) The Author(s) 2018

\begin{abstract}
Fatigue has a large impact on quality of life and is still unmanageable for many patients. Study aims were describe (1) the prevalence and pattern of fatigue over time in patients with early rheumatoid arthritis under a treat-to-target strategy and (2) identify predictive factors for worsening and recovering of fatigue over time. Data from the tREACH study were used, comparing different treatment strategies with fatigue as secondary objective. Patient outcomes on fatigue, quality of life, depression, and coping were obtained every 6 months and clinically assessed every 3 months. Prediction of fatigue at 12 months was investigated with an ROC curve. Analysis was stratified into non-fatigue and fatigue at baseline. Logistic regression was used for the evolution of fatigue in relation with the covariates over time. Almost half of all patients $(n=246)$ had high fatigue levels at baseline, decreasing slightly over time. At 12 months, $43 \%$ of patients were fatigued; while $23 \%$ of the initially fatigued patients showed lower levels of fatigue, the fatigue level had increased in $15 \%$ of the initially non-fatigued patients. The strongest predictor of fatigue was the previous fatigue levels (AUC 0.89). Higher score on the depression scale and coping with limitations was associated with developing fatigue over time in the initially non-fatigued group. Despite a strict treat-to-target strategy, fatigue remained an overall problem during the first year of treatment, and was mainly predicted by its baseline status. In subgroups, a small additional effect of depression was seen. Monitoring fatigue and depression may be important in managing fatigue.
\end{abstract}

Keywords Early RA $\cdot$ Fatigue $\cdot$ Patient-reported outcomes

\section{Background}

Studies have shown good results concerning remission and structural radiographic damage of the joints by tight control and treat-to-target management $[1,2]$. Despite these effective strategies and reaching remission of disease activity, patients with rheumatoid arthritis (RA) still may experience a burden of the diseases like pain and fatigue [3]. From 40 to $80 \%$ of the RA patients are fatigued, which may affect their lives [4-6]. The impact of fatigue permeates through every aspect of their lives, limiting work participation [7],

Margot J. M. Walter

m.walter@erasmusmc.nl

1 Department of Rheumatology, Erasmus Medical Center, University Medical Center Rotterdam, Postal box 2040, 3000 CA Rotterdam, The Netherlands

2 Department of Rheumatology, Maasstad Hospital, Rotterdam, The Netherlands family activities or social activities [8], sports and simply enjoying life as it is.

Thus, fatigue is an important aspect for many patients with a high impact on patients by influencing the choices they make in their social life. Moreover, fatigue is associated with a reduced health-related quality of life and depression $[8,9]$ and is the most limiting factor for the ability to work [10]. Because of this large impact, it is important to study fatigue. So far, little is known about fatigue during the disease course in early RA. According to patients, reducing fatigue is an important treatment target, but is not often addressed during consultations [11, 12].

The evolvement of fatigue over time in patients with early RA had been addressed in a few previous studies. An 8-year study from The Netherlands suggested little change of fatigue levels over time at group level, while individual levels fluctuated over time [13]. In contrast, a study in early RA revealed an improvement in fatigue for $40 \%$ of the patients, while fatigue levels increased in another $24 \%$ [14]. These 
were both cohort studies in which treatment was left to the discretion of the physicians.

Therefore, the objective of the present study was (1) to describe the prevalence and pattern of the fatigue over time in patients with early RA under a treat-to-target strategy and (2) to identify predictive factors for worsening and recovering of fatigue over time.

\section{Methods}

\section{Study participants}

Data from the tREACH study (treatment in the Rotterdam Early Arthritis Cohort, 2007-2013), comparing different treatment strategies with fatigue as secondary objective, and patients fulfilling the ACR-EULAR 2010 criteria for RA, were used for this analysis [15]. This multi-centered trial compared different initial treatment strategies in early RA patients. Inclusion criteria for the tREACH study were: age $\geq 18$ years, arthritis in one or more joint(s) and symptom duration $<1$ year. Patients were recruited from the outpatient clinics of all participating centres between July 2007 and April 2011. Initial treatment arms were: I methotrexate, sulfasalazine, and hydroxychloroquine (HCQ) + glucocorticosteroids (GCs) intramuscularly; II methotrexate, sulfasalazine (SASP), and HCQ + oral GC-tapering scheme; and III $\mathrm{MTX}+$ oral GC. Treatment was escalated to biologicals if DAS44 > 2.4 [16-18]. Details can be found in Claessen et al. [19]. The medical ethics committee at each participating center approved the study protocol and all patients gave written informed consent before inclusion (METC 2006-252, trial protocol number 2006-005771-18).

\section{Data collection}

Patients' demographic and clinical characteristics as well as the frequency of erosions were recorded at baseline. Disease activity measures and adjustments to treatments were applied every 3 months. Fatigue, coping strategies for pain and physical limitations, health-related quality of life, and symptoms of anxiety and depression were assessed every 6 months.

\section{Clinical evaluation of disease activity}

The disease activity was assessed by the disease activity score (DAS28), which is a composite score assessing swollen joints, tender joints, and the erythrocyte sedimentation rate (ESR), and includes a Visual Analog Scale (VAS) global (range 0-10). Higher score indicates a higher disease activity [20]. To investigate the relation with fatigue and painful joints, we used the separated tender joint count (TJC 44).

\section{Patient-reported outcome measures}

\section{Fatigue}

Fatigue level was measured by VAS and the Fatigue Assessment Scale (FAS). The VAS (100 mm) fatigue involves the severity of the fatigue over the past week with the anchors: no fatigue $(0 \mathrm{~mm})$ and extremely fatigued (100 $\mathrm{mm})$. The scale is sensitive to change, valid, and reliable, but no cut-off point has been determined [21, 22]. The FAS is a ten-item fatigue scale with a good internal consistency, reliability, and validity [23, 24]. Five questions reflect physical fatigue and five questions reflect mental fatigue. The instruction is directed at how a person usually feels. Each item is scored on a five-point rating scale ranging from 1 'never' to 5 'always'. The total scores thus range from 10 to 50 and are interpreted as follows: $10-21$ no fatigue; $\geq 22-34$ substantial fatigue; and $\geq 35-50$ extreme fatigue $[25,26]$.

\section{Disease-related}

The Rheumatoid Arthritis Disease Activity Index (RADAI) measures self-reported disease activity [27]. It contains five items: global disease activity during the last month, today's disease activity in terms of swollen and tender joints, and today's severity of arthritis pain and stiffness and selfassessed tender joints. It is measured on a scale ranging from 0 to 10 , where higher scores indicate more disease activity [28].

\section{General health}

The health-related quality of life (HRQOL) was scored with the SF-36 (range 0-100). A higher score indicates a better HRQOL. It assesses eight health concepts: physical functioning, bodily pain, role limitations due to physical health problems, role limitations due to personal or emotional problems, emotional well-being, social functioning, energy/ fatigue, and general health perceptions which are summarized in a physical component summary (PCS) and mental component summary (MCS) score [29].

\section{Psychosocial}

Coping was measured via the Coping with Rheumatoid Stressors (CORS) scale. The subscales dealing with pain, decreasing activities (range 8-32), and limitations (range 10-40) were included in tREACH study. A higher sum score indicates more frequent use of the coping strategy. Both 
subscales have good internal consistency and high test-retest reliability [30, 31].

Depression and anxiety were measured by the Hospital Anxiety and Depression Scale (HADS). Two subscales with each seven items are calculated with higher scores indicating more severe symptoms of anxiety or depression [32]. Categorical scores are available. Scores between 0 and 7 represent 'no case'; 8-10 'possible case'; and 11-21 'probable case of anxiety or depression' [32, 33].

\section{Statistical analysis}

Simple descriptive techniques were used to describe the prevalence of fatigue and its associations with other covariates at baseline. Mean and SD or percentages were described, as appropriate. As longitudinal fatigue evolvement was diverse, we stratified the analysis into two clinically relevant patient samples: those with no fatigue (FAS values 10-21) and those with fatigue (FAS values 22-50) at baseline $[25,26]$. The baseline differences between fatigued and non-fatigued patients among continuous variables were tested with the unpaired $t$ test or Mann-Whitney $U$ test as appropriate. Categorical variables were tested using Pearson's Chi-square test.

Prediction of fatigue at 12 months was investigated with an ROC curve with fatigue as a continuous variable. To investigate variables that are important for change of fatigue over time, logistic regression analyses predicting fatigue status at 12 months by baseline covariates were performed for each stratum. First, univariable analyses were performed. Thereafter, starting with full models, backward elimination was performed until all remaining variables reached a significance level of $p<0.10$. Age and gender were forced into the models regardless of their levels of significance. Missing values were imputed by multiple imputation with chained equations using $m=100$ imputation data sets. $p$ values $<0.05$ were considered statistically significant.

\section{Results}

Baseline fatigue data were available for 246-270 individuals participating in the tREACH trial. The mean age was 53 years (SD 14.3 years), the DAS score was 4.8 , and $67 \%$ were females (see Table 1). At baseline, rheumatoid factor and anti-CCP antibodies were present in 73 and 77\% of patients, respectively. Erosions were present in $18 \%$ of patients (Table 1). The 24 patients that missed their baseline fatigue level worked less often $(p=0.05)$, but did not differ with respect to DAS score $(p=0.7)$, the presence of erosions $(p=0.76)$, or the treatment arm that they had been randomized to (I vs. II $p=0.79$; I vs. III $p=0.90$; II vs. III $p=0.88)$ (data not shown).

\section{Prevalence and pattern of fatigue}

At baseline, the mean VAS fatigue score was 51 (SD 26); and the mean FAS score was 22 (SD 7) and $45 \%$ of the patients were categorized as fatigued (FAS $>21$ ).

Table 1 summarizes the baseline results for all patients and broken down for the 113 fatigued and 133 non-fatigued patients. Fatigue was most commonly present in younger females. The two fatigue groups differed in disease-related characteristics and patient-reported outcomes (Table 1). Of note, $32 \%$ of the fatigued patients reached the cut point of 8 in the HADS that bears clinical relevant levels for depression, compared to $9 \%$ of the non-fatigued patients.

Over time, the FAS fatigue score on average decreased slightly, by 1.4 points in all patients and by 3.8 points in the fatigued patients, while it increased by 0.8 points in the nonfatigued patients (Fig. 1). At 12 months, $43 \%$ of all patients were still fatigued. Individual patient profiles showed varying patterns.

Of all fatigued patients $(n=113)$ at baseline, the fatigue level decreased to below the level of no fatigue in only $23 \%$, while $15 \%$ of the non-fatigued patients $(n=133)$ became fatigued.

\section{Predicting fatigue}

The strongest predictor of fatigue was the previous fatigue levels. In an area under curve (AUC) model, baseline fatigue predicted fatigue over time with an AUC of 0.89 (Fig. 2). Adding other variables did not improve the model.

\section{Factors associated with the strata of fatigue at 12 months}

The univariable analysis of patients stratified by baseline fatigue status showed a significantly higher VAS global score in the no fatigue group, as well as a lower score on the Mental Component summary of the SF-36, higher scores on the HADS depression and anxiety, more painful joints, and higher levels of DAS. In the multivariable analysis, only a higher HADS depression score and higher scores on coping with limitations were associated with developing fatigue over time (Table 2).

In the fatigued patients, no factors apart from the severity of fatigue itself explained the recovery of fatigue in the univariable and multivariable analyses (Table 2).

\section{Discussion}

In this study, almost half of the early RA patients were fatigued over the first year after diagnosis, although they had been treated by an early, intensive, and tight-controlled 
Table 1 Baseline characteristics, total, high fatigued patients, and fatigue and non-fatigued patients

\begin{tabular}{|c|c|c|c|c|}
\hline$N=246$ & All patients $(n=246)$ & $\begin{array}{l}\text { Fatigued patients }(n=113 ; \\
\text { FAS } \geq 22)\end{array}$ & $\begin{array}{l}\text { No fatigued patients } \\
(n=133 ; \text { FAS }<22)\end{array}$ & $p$ \\
\hline Age, in years ${ }^{a}$ & $53.3(14.3)$ & $51.3(14.1)$ & $55.0(14.3)$ & 0.04 \\
\hline Sex, female, $(\%)$ & $68 \%$ & $75 \%$ & $62 \%$ & $0.03^{\mathrm{c}}$ \\
\hline Working status (\%) & $55 \%$ & $52 \%$ & $60 \%$ & $0.21^{\mathrm{c}}$ \\
\hline Native, Dutch (\%) & $83 \%$ & $81 \%$ & $85 \%$ & $0.35^{\mathrm{c}}$ \\
\hline Symptom duration (days) & $161.5(88.8)$ & $166.0(91.0)$ & $158.14(87.0)$ & 0.48 \\
\hline RF-positive, $\%$ & $73 \%$ & $76 \%$ & $69 \%$ & $0.01^{\mathrm{c}}$ \\
\hline ACPA-positive, $\%$ & $77 \%$ & $76 \%$ & $80 \%$ & $0.12^{\mathrm{c}}$ \\
\hline DAS28 (range $0-10)^{\mathrm{b}}$ & $4.8(4.0-5.7)$ & $4.9(4.3-6.0)$ & $4.7(3.7-5.4)$ & $0.004^{\mathrm{d}}$ \\
\hline Tender joints (range $0-44)^{\mathrm{b}}$ & $10(5-15)$ & $11(6-18)$ & $8(4-13)$ & $<0.001^{\mathrm{d}}$ \\
\hline Swollen joints (range $0-44)^{b}$ & $8(4-12)$ & $9(4-13)$ & $7(4-11)$ & $0.12^{\mathrm{d}}$ \\
\hline $\mathrm{ESR}^{\mathrm{b}}$ & $24(14-42)$ & $23(13-44)$ & $24(15-39)$ & $0.71^{\mathrm{d}}$ \\
\hline VAS global (range $0-100)^{\mathrm{b}}$ & $53(34-69)$ & $60(49-73)$ & $49(28-63)$ & $<0.001^{\mathrm{d}}$ \\
\hline VAS fatigue (range $0-100)^{\mathrm{b}}$ & $53(31-73)$ & $70(55-80)$ & $36(36-54)$ & $<0.001^{\mathrm{d}}$ \\
\hline FAS (range $10-50)^{\mathrm{b}}$ & $21(17-27)$ & $27(25-31)$ & $17(15-19)$ & $<0.001^{\mathrm{d}}$ \\
\hline RADAI (range 0-10) ${ }^{\mathrm{b}}$ & $4.1(2.8-5.5)$ & $4.7(3.3-6.0)$ & $3.6(2.3-4.8)$ & $<0.001^{\mathrm{d}}$ \\
\hline Coping pain (range $8-32)^{\mathrm{b}}$ & $15(11-19)$ & $17(14-21)$ & $13(10-16)$ & $<0.001^{\mathrm{d}}$ \\
\hline Coping limitations (range $8-40)^{\mathrm{b}}$ & $23(17-29)$ & $25(20-30)$ & $21(16-27)$ & $<0.001^{\mathrm{d}}$ \\
\hline HADS anxiety (range $0-21)^{\mathrm{b}}$ & $5(3-8)$ & $7(5-10)$ & $4(2-6)$ & $<0.001^{\mathrm{d}}$ \\
\hline HADS depression (range $0-21)^{b}$ & $4(2-7)$ & $5(4-8)$ & $2(1-4)$ & $<0.001^{\mathrm{d}}$ \\
\hline $\begin{array}{l}\text { Possible case depression [HADS-D } \geq 8 \text {, } \\
n /(\%)]\end{array}$ & $49(19.9 \%)$ & $37(32.7 \%)$ & $12(9 \%)$ & $<0.001$ \\
\hline SF-36 PCS (range $0-100)^{\mathrm{b}}$ & $39.9(35.7-44.9)$ & $37.9(33.4-41.8)$ & $42.0(37.4-45.9)$ & $<0.001^{\mathrm{d}}$ \\
\hline SF-36 MCS (range 0-100) ${ }^{\mathrm{b}}$ & $45.4(41.0-50.5)$ & $43.4(38.7-48.2)$ & $47.5(43.7-51.9)$ & $<0.001^{\mathrm{d}}$ \\
\hline SF-36 vitality (range $0-100)^{\mathrm{b}}$ & $55.0(40-70)$ & $40.0(30-50)$ & $70.0(55-80)$ & $<0.001^{\mathrm{d}}$ \\
\hline
\end{tabular}

$R F$ rheumatoid factor, $A C P A$ anti-citrullinated protein antibodies, ESR erythrocyte sedimentation rate, VAS Visual Analog Scale, FAS Fatigue Assessment Scale, RADAI Rheumatoid Arthritis Disease Activity Index, HADS Hospital Anxiety and Depression Scale; SF-36 Short Form 36, PCS physical component summary, MCS mental component summary

${ }^{\mathrm{a}}$ Mean (SD)

${ }^{\mathrm{b}}$ Median (IQR)

cPearson's Chi square

${ }^{\mathrm{d}}$ Mann-Whitney $U$ test

strategy. Of those who had no fatigue at baseline, $15 \%$ became fatigued, while most of those who were fatigued at baseline (77\%) remained fatigued despite lesser disease activity. The minor change in fatigue levels was also reflected in the AUC analysis of all patients, which showed that baseline level of fatigue was the strongest factor in predicting follow-up levels of fatigue. This factor was so strong that adding other variables did not improve the model. In a stratified analysis among the non-fatigued patients at baseline, higher levels on the HADS were associated with higher levels of fatigue later on.

The literature on the course of fatigue in early RA has been conflicting. A cohort study in early RA patients reported recovery of fatigue over time [14]. Recovery over time is more often observed in studies evaluating biological treatment with longstanding RA patients [34,35]. However, another cohort study showed persistent fatigue over time with almost no change since diagnosis [13]. Moreover, a recent meta-analysis found that treatment with biologicals only led to a small, but statistically significant, improvement in levels of fatigue [36]. Since our study was performed in an early RA population treated with induction by conventional DMARDs, it is interesting to see a similar pattern, with fatigue decreasing by only $6 \%$ over 1 year of follow-up.

At baseline, both inflammatory disease characteristics and patient-reported characteristics were more pronounced in the fatigued than in the non-fatigued patients. It is not clear; therefore, whether fatigue in the present study was related to the disease or to other more personal characteristics. Some direct and indirect observations suggest a less prominent relationship with the disease itself. Over time, disease activity decreased, while fatigue remained present in most patients. In the initially non-fatigued patients, the presence of symptoms related to depression and anxiety, 
Fig. 1 a Evolution over time of fatigue. b Individual profiles patients with no fatigue- $\mathbf{a}$ FAS; b DAS28. c Individual profiles patients with high fatigue-a FAS; b DAS28

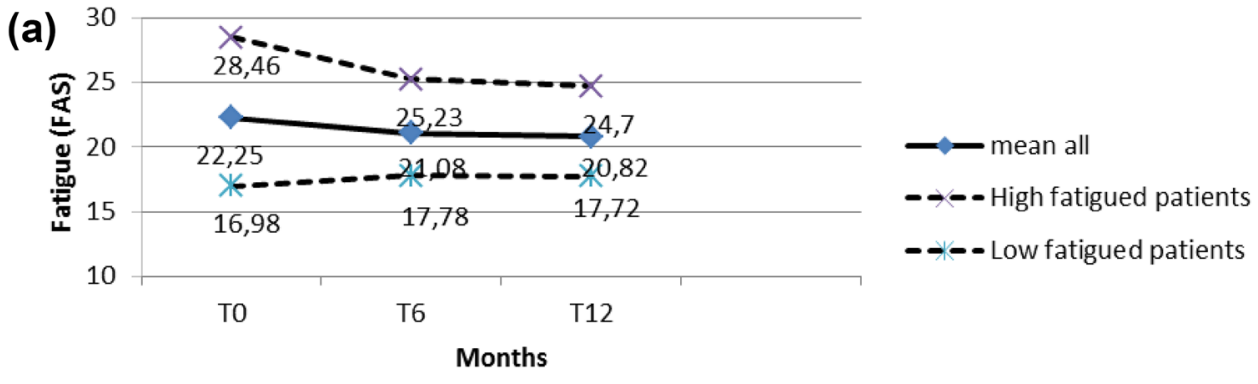

Fatigue measured with the FAS (range 10-50)

(b)

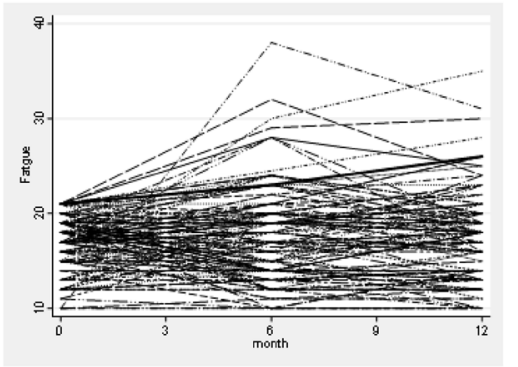

Panel A Fatigue measured with the FAS (range 10-50)

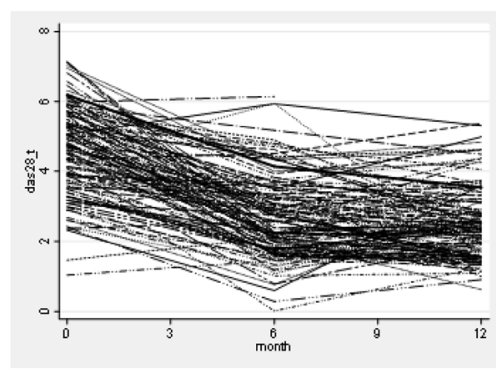

Panel B DAS28 (0-10)

(c)

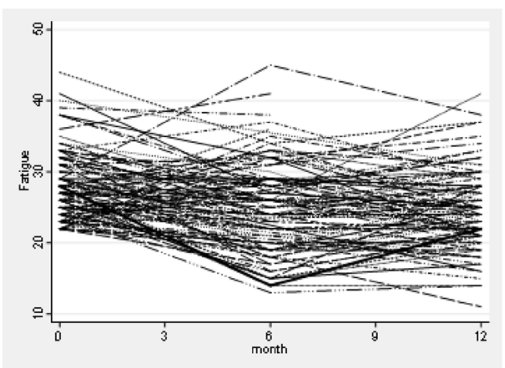

Panel A Fatigue measured with the FAS (range 10-50)

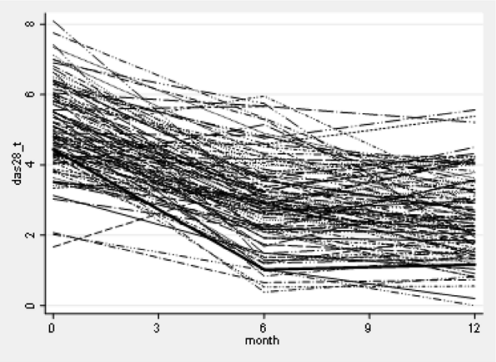

Panel B DAS28 (0-10)

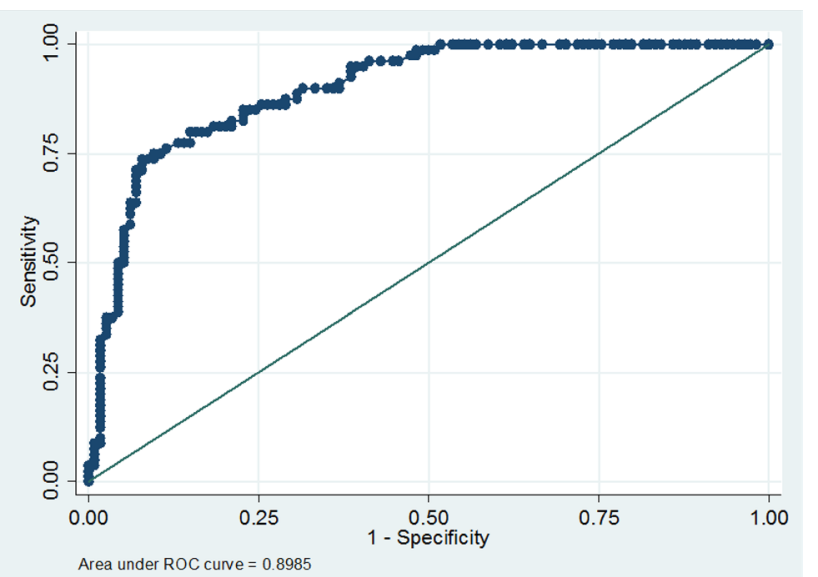

Fig. 2 ROC model with only baseline fatigue more painful joints, lower scores on the Mental Component summary of the SF-36, and higher levels of DAS were predictive in the univariate analysis for the development of fatigue. This may suggest that other pre-existing factors contribute to the presence of fatigue, of which depression/anxiety is the most powerful relation. Moreover, the relation of depression in the fatigued patients was also pronounced at baseline. Depression seems to interplay with fatigue in our early RA study population. Depressive symptoms are a common feature of both established and early RA [37] and are associated with fatigue [38]. The direction of the association, thus is depression induced by fatigue or fatigue induced by depression, is under debate. According to Druce et al., both directions are possible [39]. A dynamic conceptual model of RA fatigue showed the bi-directional relation for depression and fatigue [40]. Irrespective of the direction of this relation, the high levels of symptoms related to depression warrant monitoring 
Table 2 Univariable and multivariable analyses for developing fatigue after 12 months for low fatigued and fatigued patients

\begin{tabular}{|c|c|c|c|c|}
\hline & \multicolumn{2}{|c|}{ Univariable odds ratio $(95 \% \mathrm{CI})$} & \multicolumn{2}{|c|}{ Multivariable odds ratio $(95 \% \mathrm{CI})$} \\
\hline & Fatigue $(<21)$ & FAS $(\geq 22)$ & Fatigue $(<21)$ & FAS $(\geq 22)$ \\
\hline Sex, female & $2.60(0.83$ to 8.09$)$ & $1.28(0.48$ to 3.40$)$ & $3.01(0.84-10.73)$ & $1.83(0.65-5.01)$ \\
\hline Age, per year & 0.98 (0.95 to 1.02$)$ & $1.00(0.97$ to 1.04$)$ & $0.97(0.93-1.01)$ & $1.00(0.97-1.04)$ \\
\hline Education & $1.01(-0.03$ to 2.07$)$ & $-0.03(-1.08$ to 1.01$)$ & & \\
\hline Working status $(\mathrm{Y} / \mathrm{N})$ & $1.89(0.67$ to 5.30$)$ & $1.28(0.53$ to 3.06$)$ & & \\
\hline Nationality Natively/Dutch & $1.33(0.29$ to 6.01$)$ & $2.98(0.89$ to 9.95$)$ & $7.45(0.74-74.83)$ & $3.43(0.99-11.82)^{*}$ \\
\hline DAS28 & $1.96(1.07$ to 3.59$) *$ & $1.45(0.93$ to 2.25$)$ & & \\
\hline ESR & 1.007 (0.98 to 1.02$)$ & $1.00(0.98$ to 1.02$)$ & & \\
\hline Tender joints $(0-44)$ & $1.12(1.01 \text { to } 1.23)^{* *}$ & 1.03 (0.96 to 1.10$)$ & & \\
\hline Swollen joints (0-44) & $1.03(0.95$ to 1.12$)$ & $1.02(0.96$ to 1.08$)$ & & \\
\hline VAS global (0-100) & $1.02(1.00 \text { to } 1.05)^{* *}$ & $1.01(0.99$ to 1.04$)$ & & \\
\hline Radai (0-10) & $1.19(0.88$ to 1.61$)$ & $0.97(0.75$ to 1.25$)$ & & \\
\hline Hads depression (0-21) & $1.20(1.03 \text { to } 1.40)^{* *}$ & 1.04 (0.91 to 1.19$)$ & $1.33(1.08-1.62)^{* *}$ & \\
\hline Hads anxiety $(0-21)$ & $1.15(1.01 \text { to } 1.32)^{*}$ & 0.98 (0.86 to 1.12$)$ & & \\
\hline Coping limitations (8-40) & $1.06(0.99$ to 1.14$)$ & $0.98(0.92$ to 1.04$)$ & $1.09(1.00-1.18)^{*}$ & \\
\hline Coping pain (8-32) & $1.08(0.98$ to 1.19$)$ & $0.96(0.87$ to 1.05$)$ & & \\
\hline Physical health (SF-36, 0-100) & $0.94(0.86$ to 1.02$)$ & $1.00(0.94$ to 1.07$)$ & & \\
\hline Mental health (SF-36, 0-100) & $0.87(0.80 \text { to } 0.96)^{* *}$ & $0.98(0.92$ to 1.05$)$ & & \\
\hline
\end{tabular}

Multivariable analysis corrected for sex and age. Level of significance $* p=0.05 / * * p=0.01 / * * * p=0.001$ cut point for FAS $\leq 21$ non-fatigue $>22$ fatigued

ESR erythrocyte sedimentation rate, VAS Visual Analog Scale, RADAI Rheumatoid Arthritis Disease Activity Index, HADS Hospital Anxiety and Depression Scale, SF-36 Short Form 36, PCS physical component summary, MCS mental component summary

over time and further examination by a psychologist if symptoms persist.

Several aspects of this study need further discussion. There are many ways to analyse fatigue over time. We used simple logistic regression and the AUC, but also considered longitudinal models taking into account individual patient profiles. These models did not lead to different results and insights than described in the analysis presented here.

In 2003, it had been decided to use the FAS and the VAS fatigue, at a time when not many specific RA fatigue instruments were available. The FAS has a good internal consistency reliability and validity [24, 25]. The lack of a standardized VAS cut-off point for high and low fatigue prevented a clear interpretation of the VAS fatigue scores. We were able to analyse a substantial number of covariates influencing fatigue, but data on, for example, sleep quality or the presence of symptoms of fibromyalgia were not available. Strong points of this study include its longitudinal design and the use of data of the protocolled medication and tight-controlled treatment. As this study was not an RCT, we could study the longitudinal evolvement of fatigue and the development of fatigue among those patients with initial low fatigue level at baseline and recovery of fatigue among those with initial high level of fatigue. Medication had no effect on the decrease of fatigue in both groups (data not shown).
Given that many patients in this study in early RA showed fatigue, it seems advisable to quantify fatigue and depression in daily care. Our results and those of others suggest that fatigue does not resolve by itself. To facilitate (more) self-management behaviour, it is important to inform patients about the course of fatigue. Nurses are ideally suited to address this topic during consultations.

Screening on fatigue and depressive symptoms at baseline and follow-up will make a patient feel that his or her fatigue is acknowledged, and may also improve patient satisfaction and treatment outcomes.

\section{Conclusion}

Despite a strict treat-to-target strategy in early RA patients, fatigue is and remains a problem for many patients. Initial fatigue level is the main predictor for fatigue at follow-up. Higher levels of depression were associated with developing fatigue in initially non-fatigued patients. Discussing and monitoring fatigue and depression at baseline and follow-up might be important to acknowledge its presence and improve patients' self-management of fatigue. Referral to a psychologist may be indicated. 
Acknowledgements We thank all patients who are enrolled in the tREACH trial. Without their active cooperation, our trial would not be possible. The tREACH trial comprises the following rheumatology centres: Erasmus MC, Rotterdam; Sint Franciscus Gasthuis, Rotterdam; Maasstad Ziekenhuis, Rotterdam; Vlietland Ziekenhuis, Schiedam; Admiraal de Ruyter Ziekenhuis, Goes and Vlissingen; Zorgsaam Ziekenhuis, Terneuzen; Albert Schweitzer Ziekenhuis, Dordrecht.

Author contributions MW, TK, JH, and JL were involved in the study conceptualization and design; MW, TK, and JL performed the analysis; all authors participated in the interpretation of the data; MW prepared the first draft of the manuscript; all authors read, contributed, and approved the final version of the manuscript; and MW is responsible for the integrity of the work and is the corresponding author.

Funding Not obtained for this study.

\section{Compliance with ethical standards}

Conflict of interest All authors declare no conflicts of interest.

Ethical approval The medical ethics committee at each participating center approved the study (METC 2006-252, trial protocol number 2006-005771-18).

Human and animal rights All procedures performed in studies involving human participants were in accordance with the ethical standards of the institutional and with the 1964 Helsinki declaration and its later amendments or comparable ethical standards.

Informed consent Informed consent was obtained from all individual participants included in the study.

Open Access This article is distributed under the terms of the Creative Commons Attribution 4.0 International License (http://creativeco mmons.org/licenses/by/4.0/), which permits unrestricted use, distribution, and reproduction in any medium, provided you give appropriate credit to the original author(s) and the source, provide a link to the Creative Commons license, and indicate if changes were made.

\section{References}

1. Haugeberg G, Boyesen P, Helgetveit K, Proven A (2015) Clinical and radiographic outcomes in patients diagnosed with early rheumatoid arthritis in the first years of the biologic treatment era: a 10-year prospective observational study. J Rheumatol 42(12):2279-2287

2. Nieuwenhuis WP, de Wit M, Boonen A, van der Helm-van Mil A (2016) Changes in the clinical presentation of patients with rheumatoid arthritis from the early 1990s to the years 2010: earlier identification but more severe patient reported outcomes. Ann Rheum Dis 75(11):2054-2056

3. Fautrel B, Alten R, Kirkham B, de la Torre I, Durand F, Barry J, Taylor PC (2018) Call for action: how to improve use of patient-reported outcomes to guide clinical decision making in rheumatoid arthritis. Rheumatol Int 38:935-947

4. Belza BL, Henke CJ, Yelin EH, Epstein WV, Gilliss CL (1993) Correlates of fatigue in older adults with rheumatoid arthritis. Nurs Res 42(2):93-99
5. Pollard LC, Choy EH, Gonzalez J, Khoshaba B, Scott DL (2006) Fatigue in rheumatoid arthritis reflects pain, not disease activity. Rheumatology 45(7):885-889

6. Wolfe F, Hawley DJ, Wilson K (1996) The prevalence and meaning of fatigue in rheumatic disease. $J$ Rheumatol 23(8):1407-1417

7. Mancuso CA, Rincon M, Sayles W, Paget SA (2006) Psychosocial variables and fatigue: a longitudinal study comparing individuals with rheumatoid arthritis and healthy controls. J Rheumatol 33(8):1496-1502. doi:06/13/0618

8. Matcham F, Ali S, Hotopf M, Chalder T (2015) Psychological correlates of fatigue in rheumatoid arthritis: a systematic review. Clin Psychol Rev 39:16-29

9. Nikolaus S, Bode C, Taal E, van der Laar M (2013) Fatigue and factors related to fatigue in rheumatoid arthritis: a systematic review. Arthritis Care Res 65(7):1128-1146

10. Lacaille D, White MA, Backman CL et al (2007) Problems faced at work due to inflammatory arthritis: new insights gained from understanding patients' perspective. Arthritis Care Res 57:1269-1279

11. Taylor PC, Moore A, Vasilescu R, Alvir J, Tarallo M (2016) A structured literature review of the burden of illness and unmet needs in patients with rheumatoid arthritis: a current perspective. Rheumatol Int 36(5):685-695

12. van Tuyl LHD, Boers M (2017) Rheumatoid arthritis: remission-keeping the patient experience front and centre. Nat Rev Rheumatol 13(10):573-574

13. van Steenbergen HW, Tsonaka R, Huizinga TW, Boonen A, van der Helm-van Mil AH (2015) Fatigue in rheumatoid arthritis; a persistent problem: a large longitudinal study. RMD Open 1(1):e000041

14. Rat AC, Pouchot J, Fautrel B, Boumier P, Goupille P, Guillemin F (2012) Factors associated with fatigue in early arthritis: results from a multicenter national French cohort study. Arthritis Care Res (Hoboken) 64(7):1061-1069

15. Aletaha D, Neogi T, Silman AJ, Funovits J, Felson DT, Bingham CO et al (2010) 2010 rheumatoid arthritis classification criteria: an American College of Rheumatology/European League Against Rheumatism collaborative initiative. Ann Rheum Dis 69:1580 1588. https://doi.org/10.1136/ard.2010.138461

16. de Jong PH, Hazes JM, Barendregt PJ, Huisman M, van Zeben D, van der Lubbe PA, Gerards AH, de Jager MH, de Sonnaville PB, Grillet BA, Luime JJ, Weel AE (2013) Induction therapy with a combination of DMARDs is better than methotrexate monotherapy: first results of the tREACH trial. Ann Rheum Dis 72(1):72-78

17. de Jong PH, Hazes JM, Han HK, Huisman M, van Zeben D, van der Lubbe PA, Gerards AH, van Schaeybroeck B, de Sonnaville PB, van Krugten MV, Luime JJ, Weel AE (2014) Randomised comparison of initial triple DMARD therapy with methotrexate monotherapy in combination with low-dose glucocorticoid bridging therapy; 1-year data of the tREACH trial. Ann Rheum Dis 73(7):1331-1339

18. de Jong PH, Hazes JM, Buisman LR, Barendregt PJ, van Zeben $\mathrm{D}$, van der Lubbe PA, Gerards AH, de Jager MH, de Sonnaville PB, Grillet BA, Luime JJ, Weel AE (2013) Best cost-effectiveness and worker productivity with initial triple DMARD therapy compared with methotrexate monotherapy in early rheumatoid arthritis: cost-utility analysis of the tREACH trial. Ann Rheum Dis 72(1):72-78. https://doi.org/10.1136/annrheumdis-2011-201162 (Epub 2012 Jun 7)

19. Claessen SJ, Hazes JM, Huisman MA, van Zeben D, Luime JJ, Weel AE (2009) Use of risk stratification to target therapies in patients with recent onset arthritis; design of a prospective randomized multicenter controlled trial. BMC Musculoskelet Disord 10:71 
20. van der Heijde DM, van 't Hof M, van Riel PL, van de Putte LB (1993) Development of a disease activity score based on judgment in clinical practice by rheumatologists. J Rheumatol 20(3):579-581

21. Hewlett S, Dures E, Almeida C (2011) Measures of fatigue: Bristol Rheumatoid Arthritis Fatigue Multi-Dimensional Questionnaire (BRAF MDQ), Bristol Rheumatoid Arthritis Fatigue Numerical Rating Scales (BRAF NRS) for severity, effect, and coping, Chalder Fatigue Questionnaire (CFQ), Checklist Individual Strength (CIS20R and CIS8R), Fatigue Severity Scale (FSS), functional assessment chronic illness therapy (Fatigue) (FACIT-F), multi-dimensional assessment of fatigue (MAF), multi-dimensional fatigue inventory (MFI), pediatric quality of life (PedsQL) multi-dimensional fatigue scale, profile of fatigue (ProF), Short Form 36 Vitality Subscale (SF-36 VT), and Visual Analog Scales (VAS). Arthritis Care Res (Hoboken) 63(Suppl 11):S263-S286

22. Wolfe F (2004) Fatigue assessments in rheumatoid arthritis: comparative performance of Visual Analog Scales and longer fatigue questionnaires in 7760 patients. J Rheumatol 31(10):1896-1902

23. Michielsen HJ, De Vries J, Van Heck GL (2003) Psychometric qualities of a brief self-rated fatigue measure: the Fatigue Assessment Scale. J Psychosom Res 54(4):345-352

24. De Vries J, Michielsen H, Van Heck G, Drent M (2004) Measuring fatigue in sarcoidosis: the Fatigue Assessment Scale (FAS). Br J Health Psychol 9(Pt 3):279-291

25. De Vries J, Rothkrantz-Kos S, van Dieijen-Visser MP, Drent M (2004) The relationship between fatigue and clinical parameters in pulmonary sarcoidosis. Sarcoidosis Vasc Diffus Lung Dis 21(2):127-136

26. Michielsen HJ, Drent M, Peros-Golubicic T, De Vries J (2006) Fatigue is associated with quality of life in sarcoidosis patients. Chest 130(4):989-994

27. Fransen J, Langenegger T, Michel BA, Stucki G (2000) Feasibility and validity of the RADAI, a self-administered Rheumatoid Arthritis Disease Activity Index. Rheumatology 39(3):321-327

28. Fransen J, van Riel PL (2009) Outcome measures in inflammatory rheumatic diseases. Arthritis Res Ther 11(5):244

29. Ware JE Jr, Sherbourne CD (1992) The MOS 36-item short-form health survey (SF-36). I. Conceptual framework and item selection. Med Care 30(6):473-483
30. van Lankveld W, Naring G, van 't Pad Bosch P, van de Putte L (1999) Behavioral coping and physical functioning: the effect of adjusting the level of activity on observed dexterity. J Rheumatol 26(5):1058-1064

31. van Lankveld W, van't Pad Bosch P, van de Putte L, Naring G, van der Staak C (1994) Disease-specific stressors in rheumatoid arthritis: coping and well-being. Br J Rheumatol 33(11):1067-1073

32. Zigmond AS, Snaith RP (1983) The Hospital Anxiety and Depression Scale. Acta Psychiatr Scand 67(6):361-370

33. Bjelland I, Dahl AA, Haug TT, Neckelmann D (2002) The validity of the Hospital Anxiety and Depression Scale. An updated literature review. J Psychosom Res 52(2):69-77

34. Gossec L, Steinberg G, Rouanet S, Combe B (2015) Fatigue in rheumatoid arthritis: quantitative findings on the efficacy of tocilizumab and on factors associated with fatigue. The French multicentre prospective PEPS Study. Clin Exp Rheumatol 33(5):664-670

35. Rigby W, Ferraccioli G, Greenwald M, Zazueta-Montiel B, Fleischmann R, Wassenberg S, Chen A (2011) Effect of rituximab on physical function and quality of life in patients with rheumatoid arthritis previously untreated with methotrexate. Arthritis Care Res (Hoboken) 63(5):711-720

36. Almeida C, Choy EH, Hewlett S, Kirwan JR, Cramp F, Chalder T, Christensen R (2016) Biologic interventions for fatigue in rheumatoid arthritis. Cochrane Database Syst Rev 6:CD00833

37. Schieir O, Thombs BD, Hudson M, Taillefer S, Steele R, Berkson L, Bertrand C, Couture F, Fitzcharles M (2009) Symptoms of depression predict the trajectory of pain among patients with early inflammatory arthritis: a path analysis approach to assessing change. J Rheumatol 36(2):231-239

38. Nikolaus S, Bode C, Taal E, van de Laar MA (2013) Fatigue and factors related to fatigue in rheumatoid arthritis: a systematic review. Arthritis Care Res (Hoboken) 65(7):1128-1146

39. Druce KL, Jones GT, Macfarlane GJ, Basu N (2015) Determining pathways to improvements in Rheumatoid Arthritis fatigue: results from the BSRBR-RA. Arthritis Rheumatol 67:2303-2310

40. Hewlett S, Chalder T, Choy E, Cramp F, Davis B, Dures E, Kirwan J (2011) Fatigue in rheumatoid arthritis: time for a conceptual model. Rheumatology 50(6):1004-1006 